\title{
COMIDA CHATARRA Y OBESIDAD: UN DULCE PERO NADA FELIZ CAMINO A LA DIABETES
}

\author{
JUNK FOOD AND OBESITY: \\ A SWEET, FAR FROM HAPPY ROAD TO DIABETES
}

Haciendo tiempo en un aeropuerto a mediados del pasado junio, aburrido de las lecturas habituales, decidí distraerme con un ejemplar de la revista de la National Geographic Society. Imprevistamente desde el índice me capturó el título de un artículo escrito por Tracie McMillan', una periodista independiente de los Estados Unidos, que trata temas relacionados con alimentación y equidad. El artículo se titulaba: "Do corn subsidies really make us fat?" (algo así como: "¿Es cierto que los subsidios al maíz nos engordan?"), y tomaba como punto de partida un trabajo científico publicado en 2016 en la revista JAMA Internal Medicine por un grupo de autores pertenecientes, entre otras instituciones, a los Centros de Control y Prevención de Enfermedades (CDC, sus siglas en inglés) de la ciudad de Atlanta. Claro está que, aún no finalizada la lectura del mismo, no me quedó otro remedio que volver al viejo y conocido PubMed para buscar el trabajo en cuestión, encabezado por la Dra. Karen R. Siegel, epidemióloga de la División Diabetes del Centro Nacional de Prevención de Enfermedades Crónicas y Promoción de la Salud de los CDC², con lo cual mi intento de diversificar la lectura entre avión y avión fracasó rotundamente.

McMillan relata en su artículo periodístico que Siegel et al., a partir de una encuesta alimentaria realizada entre 2001 y 2006, calcularon qué porcentaje de las calorías de sus dietas provenía de alimentos originados en productos agropecuarios subsidiados por el gobierno, incluyendo maíz, soja, trigo, arroz, leche y carnes, y encontraron una asociación significativa entre la cantidad de calorías aportada por alimentos originados en estos productos agropecuarios y distintos factores de riesgo cardiovascular. La periodista aclara que estos productos agrope- cuarios, que podrían eventualmente ser considerados saludables si se ingieren en forma no procesada, llegan a los consumidores transformados en alimento balanceado para los feedlots donde se originan productos cárnicos con un alto contenido de grasas saturadas, o en bebidas con edulcorantes ricos en calorías (como el jarabe de maíz con alto contenido en fructosa o JMAF), o en productos de panadería ricos en harinas, grasas y JMAF.

La periodista señala además que existe una contradicción entre las recomendaciones alimentarias emanadas de las autoridades de salud de los Estados Unidos y la política de subsidios que termina por incrementar la disponibilidad y facilitar el acceso a alimentos no saludables (la relación calculada entre el monto de los subsidios destinados a estimular la producción frutihortícola y el de aquellos que terminan generando comida chatarra en aquel país es de aproximadamente 1:30).

Obviamente entre los comentarios que los lectores hacían sobre el artículo de la National Geographic se retomaba el debate sobre el rol del JMAF en la génesis de la epidemia de obesidad en los Estados Unidos. Un sinnúmero de trabajos científicos, tanto epidemiológicos como experimentales, así como estudios clínicos prospectivos, han asociado a la fructosa, sola o combinada con glucosa (en forma de sacarosa o en el JMAF), con distintas alteraciones, tanto metabólicas (obesidad, insulinorresistencia, dislipoproteinemia, diabetes mellitus, hiperuricemia, esteatosis hepática no alcohólica) como cardiovasculares (hipertensión arterial) y renales ${ }^{3-7}$. Las consecuencias de medidas como el bloqueo comercial a Cuba (que en la década de 1960 cortó la principal fuente de sacarosa de bajo costo) y la mencio- 
nada política de subsidios (que destinó, en los últimos 20 años, casi 5 mil millones de dólares anuales) dirigida fundamentalmente a los productores que siembran grandes extensiones de maíz, no han hecho más que poner en manos (o en la boca) de los consumidores el tan anhelado sabor dulce a un bajo precio para el bolsillo pero a un alto costo para la salud. El JMAF obtenido a partir del maíz subsidiado es barato y presenta una serie de ventajas con respecto a la sacarosa en cuanto a gusto, conservación y manipulación. Obviamente esto resulta en una mayor disponibilidad de alimentos dulces, pero sobre todo, de bebidas con alto contenido de fructosa, que están a la venta a toda hora y todos los días de la semana (en estaciones de servicio y farmacias o convenience stores, por ejemplo) y cuya ingesta se asocia a un bajo poder de saciación ${ }^{8}$.

El debate se mantiene, en gran parte, gracias a una estrategia ya empleada por las tabacaleras (puesta de manifiesto por la Organización Mundial de la Salud (OMS) en su página: www.who.int/tobacco/media/en/ TobaccoExplained.pdf): subvencionar estudios "científicos" que niegan o mitigan las consecuencias adversas sobre la salud que genera el consumo de cantidades excesivas de fructosa contenida, fundamentalmente, en bebidas elaboradas con JMAF. Un claro ejemplo de esta práctica surge de la lectura de una interesante "revisión de revisiones" publicada en 2013 en PLOS Medicine ${ }^{9}$, en la que Bes-Rastrollo et al. -al analizar 18 revisiones sistemáticas que estudiaban la asociación entre consumo de bebidas azucaradas y el aumento de peso publicadas en los últimos 40 años en tres bases de datos (PubMed, Cochrane y Scopus)- encontraron un llamativo sesgo: mientras que, en su gran mayoría $(>80 \%$ ) las revisiones realizadas por científicos que declaraban no tener conflictos de intereses con las industrias involucradas encontró una asociación positiva entre consumo de estas bebidas y el aumento de peso, una proporción similar de aquellos científicos que declaraba tener alguna vinculación económica con la industria de los alimentos concluyó que la evidencia científica era insuficiente como para establecer fehacientemente dicha asociación. A partir de estas proporciones puede establecerse que es cinco veces más probable que un estudio publicado por investigadores que declaran tener conflictos de interés niegue la asociación entre consumo de bebidas azucaradas y aumento de peso ( $R R=5,0,95 \% \mathrm{Cl}$ : 1,3-19,3).

Resulta importante señalar que, en el trabajo publicado por Siegel et al. ${ }^{2}$, la proporción de calorías provenientes de comida chatarra era significativamente superior en los sujetos más jóvenes. En este sentido Bowman et al. ${ }^{10}$ evaluaron patrones de ingesta de niños y adolescentes ( $n \sim 6200$, edad 4-19 años) y hallaron que, en un día promedio cualquiera, un 30,3\% consumía algún tipo de comida chatarra, y que esta ingesta se relacionaba con un incremento significativo en el aporte calórico (más grasas saturadas, más azúcares simples), y menos leche, vegetales y fibra alimentaria (un claro ejemplo de malnutrición). La obesidad y la dislipoproteinemia resultantes de la ingesta de azúcares simples agregados a la dieta se asocian, de acuerdo a un documento reciente de la American Heart Association, a un incremento del riesgo cardiovascular en esta población ${ }^{11}$. También en estos grupos etarios se ha demostrado que la exposición aguda a la publicidad de alimentos aumenta en forma significativa la ingesta ${ }^{12}$, lo que resalta la importancia de tomar medidas destinadas a minimizar la exposición de los niños a campañas que publicitan alimentos no saludables.

Como parte de la estrategia orientada a reducir el impacto de las enfermedades crónicas no transmisibles, la OMS instó en 2010 a la comunidad internacional para, entre otras cosas, limitar la exposición de los niños al marketing de alimentos ricos en grasas saturadas, ácidos grasos trans, azúcares simples o sal. Un informe reciente de la Organización Panamericana de la Salud (OPS, http://iris.paho.org/xmlui/bitstream/ handle/123456789/7698/9789275318645_esp. pdf) señala que en América Latina se ha registrado en un fuerte incremento del consumo de este tipo de alimentos ultraprocesados (nueva- 
mente una estrategia poco original, que copia lo ocurrido décadas atrás ante el fuerte descenso del consumo de tabaco en los "países centrales") y lo vincula a la tendencia creciente en la prevalencia de obesidad. En nuestro país la ANMAT, por su disposición 6516/2015, establece una reglamentación para las características de la publicidad de alimentos; entre los fundamentos se advierte la preocupación por “...garantizar que la información provista sea veraz y objetiva, con el fin de que no induzca a errores de comprensión ni dé lugar a promociones engañosas que predispongan a conductas y/o hábitos no saludables...", así como de "... garantizar que la información brindada sea (...) veraz, precisa y clara, con el fin de propender a la utilización adecuada del producto, presentando sus propiedades objetivamente sin engaños o equívocos." Esto, lamentablemente, es un objetivo que dista de cumplirse. Resulta imperativo, además, contrarrestar el multimillonario y malintencionado marketing de la comida -y la bebida- chatarra mediante campañas incisivas de educación nutricional en los colegios (se encuentran en tratamiento en el Poder Legislativo de la Nación dos proyectos en este sentido) para así empoderar a niños y jóvenes, y a través de ellos, a sus familias, para que no se asocien más estos hábitos alimentarios con la felicidad, sino con la enfermedad y priorizar, gracias a la concientización, una nutrición saludable.

Urge también lograr la implementación de un sistema de rotulado de los alimentos, como el que rige en Chile desde 2016: sellos claramente visibles denotan si un producto contiene, como resultado de su composición, altos niveles de sodio, azúcares simples, grasas saturadas o calorías. Otra posible herramienta legal, que ha demostrado tener un impacto sobre el consumo de productos ricos en fructosa (pero que, por lógica, es fuertemente resistida por la industria) es gravar la venta de los mismos, táctica implementada en países como México y Brasil.

Éstas y otras medidas (por ejemplo, subsidios para productores de alimentos saludables y/o desgravación de este tipo de alimentos) pueden y deben ser implementadas en un cor- to plazo. Sin una acción comunitaria tendiente a imponerlas, suena utópico pensar en frenar la creciente epidemia de obesidad y trastornos de salud relacionados.

\section{Dr. Pablo Arias}

Médico Endocrinólogo, Profesor Titular Ordinario, Cátedra de Fisiología Humana, Facultad de Ciencias Médicas, Universidad Nacional de Rosario

\section{BIBLIOGRAFÍA}

1. McMillan T. Are corn subsidies making us fat? http:// www.nationalgeographic.com/people-and-culture/ food/the-plate/2016/07/are-corn-subsidies-making-usfat-/. Consulta: 12/08/2017.

2. Siegel KR, McKeever Bullard K, et al. Association of higher consumption of foods derived from subsidized commodities with adverse cardiometabolic risk among US adults. JAMA Intern Med 2016;176(8):1124-1132.

3. Hu FB. Resolved: there is sufficient scientific evidence that decreasing sugar-sweetened beverage consumption will reduce the prevalence of obesity and obesityrelated diseases. Obes Rev 2013; 14: 606-9.

4. Stanhope KL. Role of fructose-containing sugars in the epidemics of obesity and metabolic syndrome. Annual Review of Medicine 2012; 63:329-34.

5. Gao X, Qi L, Qiao N, et al. Intake of added sugar and sugar-sweetened drink and serum uric acid concentration in US men and women. Hypertension 2007; 50: 306-312.

6. Ferder L, Ferder MD, Inserra F. The role of high-fructose corn syrup in metabolic syndrome and hypertension. Current Hypertension Reports 2010; 12: 105-12.

7. Johnson RJ, Sanchez-Lozada LG, Nakagawa T. The effect of fructose on renal biology and disease. J Am Soc Nephrol 2010; 21: 2036-9.

8. Lowette K, Roosen L, et al. Effects of high-fructose diets on central appetite signaling and cognitive function. Front Nutr 2015; 2:5.

9. Bes-Rastrollo M, Schulze MB, et al. Financial conflicts of interest and reporting Bias regarding the association between sugar-sweetened beverages and weight gain: a systematic review of systematic reviews. Plos Medicine 2013; 10: e1001578.

10. Bowman SA, Gortmaker SL, et al. Effects of fast-food consumption on energy intake and diet quality among children in a national household survey. Pediatrics 2004; 113: 112-8.

11. American Heart Association. Added sugars and cardiovascular disease risk in children: a scientific statement from the American Heart Association. Circulation 2017; 135): e1017-e1034.

12. Boyland EJ, Nolan S, et al. Advertising as a cue to consume: a systematic review and meta-analysis of the effects of acute exposure to unhealthy food and nonalcoholic beverage advertising on intake in children and adults. Am J Clin Nutr 2016; 103:519-33. 